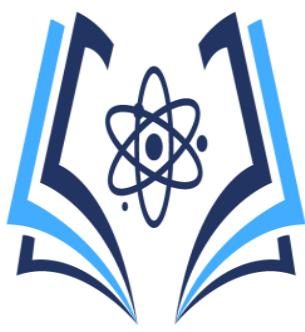

\title{
Medidas generales de prevención de enfermedad pulmonar obstructiva crónica
}

\author{
General measures for the prevention of chronic obstructive \\ pulmonary disease
}

\section{Medidas generales de prevención de enfermedad pulmonar}

María Pincay Cañarte. Mg

Universidad Estatal del Sur de Manabí, maria.pincay@unesum.edu.ec, https://orcid.org/0000-0003-4110-9275

Contacto: maria.pincay@unesum.edu.ec

Recibido: 28-09-2019

Aprobado: 15-12-2019

\section{Resumen}

La Enfermedad Pulmonar Obstructiva Crónica (EPOC) es una enfermedad inflamatoria que constituye un gran problema de salud pública y una de las principales causas de morbimortalidad en Ecuador y en el mundo. El objetivo de esta investigación fue Identificar las medidas generales de prevención de la enfermedad pulmonar obstructiva crónica. Se realizó un estudio descriptivo, sustentado en la revisión bibliográfica de investigaciones de tipo científico, relacionadas con el tema, en las cuales se consultaron diferentes fuentes de carácter nacional e internacional como Medline Plus, Clinic Barcelona, Cuídate Plus, Scielo, OMS, OPS, Ministerio de Salud Pública y otros autores. Criterios de inclusión: Se seleccionaron fuentes de investigaciones realizadas entre 2015 y 2020 es decir que no tuvieran más de cinco años de haber sido publicadas, y cuya información este enfocada en la temática a tratar. Se incluyó Google Académico, como fuente de búsqueda de información. Criterios de exclusión: Se excluyeron fuentes bibliográficas con más de 5 años de vigencia y trabajos cuya base de datos no son de libre acceso, e información de fuentes no confiables. La técnica utilizada fue la compilación bibliográfica, de documentos que publican información confiable, entre los cuales están textos, páginas web, artículos etc. Los principales resultados indican que el tabaquismo es la causa más importante para desarrollar EPOC, seguido de la exposición a otras sustancias presentes en el aire inspirado, como es el caso de la exposición ocupacional. Entre las medidas generales para prevenir o para mejorar la calidad de vida a los pacientes con EPOC están, el abandonar el tabaco, adquirir hábitos de vida saludable, como llevar una dieta sana, realizar ejercicios, vacunarse contra el neumococo y la influenza, esto ayuda a disminuir el impacto de la enfermedad pulmonar obstructiva crónica
Palabras clave: Enfermedad Pulmonar obstructiva crónica, prevalencia, factor de riesgo, morbimortalidad, tabaquismo,
Abstract
Chronic Obstructive Pulmonary Disease (COPD) is an inflammatory disease that constitutes a major public health problem and one of the main causes of morbidity and mortality in Ecuador and in the world. The objective of this research was to identify general preventive measures for chronic obstructive pulmonary disease. A descriptive study was carried out, based on the bibliographic review of scientific research related to the subject, in which different national and international sources were consulted such as Medline Plus, Clinic Barcelona, Cuídate Plus, SciELO, WHO, PAHO, Ministry of Public Health and other authors. Inclusion criteria: Research sources carried out between 2015 and 2020 were selected, that is, they had not been published more than five years ago, and whose information is focused on the topic to be addressed. Academic Google was included as a source of information search. Exclusion criteria: Bibliographic sources with more than 5 years of validity and works whose database is not freely accessible, and information from unreliable sources were excluded. The technique used was the bibliographic compilation of documents that publish reliable information, among which are texts, web pages, articles, etc. The main results indicate that smoking is the most important cause for developing COPD, followed by exposure to other substances present in inspired air, as is the case with occupational exposure. Among the general measures to prevent or to improve the quality of life of COPD patients are, quitting tobacco, acquiring healthy lifestyle habits, such as eating a healthy diet, exercising, getting vaccinated against pneumococcus and influenza, this helps reduce the impact of chronic obstructive pulmonary disease 
Key words: Chronic obstructive pulmonary disease, prevalence, risk factor, morbidity and mortality, smoking,

\section{Introducción.}

Enfermedad pulmonar obstructiva crónica (EPOC) es la denominación que recibe un conjunto de enfermedades broncopulmonares en las que se ve afectado el flujo aéreo (circulación del aire) por una limitación crónica, en la mayor parte de las ocasiones, es el resultado, a largo plazo, de la reacción de los bronquios, de los pulmones y de las arterias pulmonares a la inhalación del humo de tabaco (1)

La enfermedad pulmonar obstructiva crónica (EPOC) es el problema respiratorio de mayor prevalencia, a pesar de ser una enfermedad potencialmente prevenible. Por su elevada frecuencia, su curso clínico progresivo y sus requerimientos asistenciales constituyen un problema médico de primer orden, siendo una de las principales causas de mortalidad a nivel mundial, afectando por igual a ambos sexos, siendo el tabaquismo, la contaminación ambiental y otros factores, elementos que hacen crecer esta enfermedad con complicaciones que elevan la mortalidad

La EPOC, Según las estimaciones de la OMS (Organización Mundial de la Salud) 65 millones de personas tienen enfermedad pulmonar obstructiva crónica (EPOC) de moderada a grave, de acuerdo con el Estudio de la Carga Mundial de Morbilidad, la prevalencia de la EPOC en 2016 fue de 251 millones de casos y Se estima que 3,17 millones de muertes fueron causadas por la enfermedad en 2015 a nivel mundial (5\% de todas las muertes a nivel mundial en ese año de acuerdo con los datos de la OMS) (2)

Se prevé que las muertes totales a nivel mundial por EPOC aumenten más del $30 \%$ en los próximos 10 años, a menos que se tomen medidas urgentes para reducir los factores de riesgo subyacentes, especialmente el consumo de tabaco. La OMS pronostica que en el 2030 la EPOC será la tercera causa de muerte en todo el mundo, un $7,8 \%$ de todas las muertes y el $27 \%$ de las muertes relacionadas con el tabaco, solo superada por el cáncer $(33 \%)$ y por las enfermedades cardiovasculares (29\%) (3)

En América Latina y el Caribe la prevalencia es alta, especialmente para los hombres y las personas mayores. La incidencia de esta enfermedad (en 11 ciudades, 6 países) fue del 13,4\%, y se comprobó que la mayoría de los pacientes sufren EPOC de leve o moderada, asimismo se halló que la prevalencia aumenta con la edad en 1,75 . Otros indican que 35 de cada 1.000 hospitalizaciones en estos países se debieron a la EPOC, la mayoría de alto costo económico, y la mortalidad hospitalaria por esta enfermedad varió de $6,7 \%$ a 29,5\%. (4)

Según el estudio PLATINO (proyecto latinoamericano de investigación en obstrucción pulmonar), la enfermedad pulmonar obstructiva crónica (EPOC) se proyecta como la tercera causa de muerte en el mundo en el año 2020. La prevalencia y el impacto de esta enfermedad sobre la población se espera aumente por el envejecimiento y el alza en las tasas de tabaquismo de la población, tanto en países desarrollados como subdesarrollados. La prevalencia mundial en la población general se estima en alrededor de $1 \%$ y en mayores de 40 años en $10 \%$.

En México según estudios del INER (Instituto Nacional de Enfermedades Respiratorias) en el año 2017 la EPOC se ubicó en el cuarto lugar en la tabla de morbi-mortalidad anual, siendo su prevalencia igual entre hombres y mujeres, el factor de riesgo más frecuente fue el tabaquismo, seguido de la exposición a biomasa.

El estudio de la prevalencia de la EPOC en Colombia realizada por la fundación neumológica colombiana en el año 2014, determino que a nivel nacional 9 de cada 100 personas mayores de 40 años tenían EPOC porcentaje que se distribuyó en $8,5 \%$ en Bogotá, $6,2 \%$ en barranquilla, $8,5 \%$ en Cali, $7,9 \%$ en Bucaramanga y 13,5\% en Medellín. Según las cifras presentadas por el Departamento Administrativo Nacional de Estadística (DANE), el total de muertes ocurridas fueron alrededor de 4500.

Según la base de datos obtenidos del Instituto Nacional de Estadísticas y Censos (INEC), en el año 2012 en el Ecuador, se puede observar alrededor de 4023 egresos hospitalarios por Enfermedad Pulmonar Obstructiva Crónica. El promedio de días de estadía de 7 días; de los cuales, 91.5\% correspondían a pacientes en edades comprendidas entre 45 a 65 y más años. (5)

Estos datos también corresponden a Ecuador, pero del año 2016, obtenidos del Instituto Nacional de Estadísticas y Censos (INEC), se puede observar alrededor de 22850 egresos hospitalarios por Enfermedad Pulmonar Obstructiva Crónica, el promedio de días de estadía continúa siendo de 7 ; de los cuales, 91,5\% correspondían a pacientes en edades comprendidas entre 45 a 65 años y más, rangos de edades que sean mantenido. Esta información, muestra que esta enfermedad va en aumento.

Tomando en cuenta datos regionales en la provincia de Bolívar según datos del Instituto Nacional de Estadísticas y Censos (INEC), la incidencia de enfermedad pulmonar obstructiva crónica se constituye en un $43,5 \%$ de la población en total, los 
factores que se atribuye en un gran porcentaje es el tabaquismo $23 \%$ seguido por la exposición a biomasa $14 \%$ y otros factores en un 6,5 .

En nuestro país la EPOC alcanza una prevalencia del 4.7\%; se encuentra dentro de las principales 10 causas de discapacidad y las primeras 20 causas de mortalidad en adultos, alcanzando una tasa de 10.31 por cada 100.000 habitantes. (6) La prevalencia de EPOC en mujeres está en aumento.

\section{Materiales y métodos}

Se realizó un estudio descriptivo, ya que detalla de forma clara todos los aspectos encontrados en el proceso de investigación para el análisis de los datos recolectados, así como para describir los diferentes resultados, sustentado en la revisión bibliográfica de investigaciones de tipo científico, relacionadas con el tema, en las cuales se consultaron diferentes fuentes, entre las cuales están las bases de datos de carácter internacional y nacional como Medline Plus, Clinic Barcelona, Cuídate Plus, SciELO, OMS, OPS, Ministerio De Salud y otros autores, al realizar la búsqueda, se obtuvieron un total de 60 documentos de los cuales fueron seleccionados para la investigación 38 publicados entre 2015 y 2020.

Criterios de inclusión: Para la selección de las fuentes de información se consideró, las investigaciones no tuvieran más de cinco años de haber sido publicadas, y cuya información este enfocada en la temática a tratar. Se incluyó Google Académico, como fuente de búsqueda de información.

Criterios de exclusión: Se excluyeron fuentes bibliográficas con más de 5 años de vigencia, trabajos cuya base de datos no son de libre acceso, e información de fuentes no confiables.

Los métodos utilizados fueron los empíricos (observacional) y teóricos (analítico y sintético), porque permiten el análisis de las investigaciones mediante publicaciones indexadas en revistas científicas, seleccionando las que aportan con información actualizada y confiable para el desarrollo del tema de estudio.

La técnica utilizada fue la compilación bibliográfica, lo que permitió realizar análisis y síntesis de documentos que publican información confiable, entre los cuales están textos, páginas web, artículos etc.

\section{Resultados}

Mediante un análisis exhaustivo se logó analizar diferentes estudios, y fuentes, con la finalidad de identificar los factores que inciden esta enfermedad, así como las medidas generales para prevenir la EPOC

Factores de riesgo: La EPOC es de origen multifactorial y se desarrolla por la interacción de diferentes factores de riesgo entre los cuales están el medio ambiente.
En todo el mundo, los diferentes estudios coinciden que, el factor de riesgo más comúnmente observado para la EPOC, es el fumar tabaco, sin embargo, solo un $50 \%$ de los fumadores desarrollarán la enfermedad, el riesgo se incrementa en función de la cantidad de cigarrillos fumados, la edad de comienzo y tiempo que lleva fumando, siendo el humo del tabaco el principal causante, además es importante destacar que la EPOC afecta igual tanto a hombres como a mujeres. (7)

La $O M S$ señala que para el año 2030 EPOC será la tercera causa más importante de mortalidad y cuarta de discapacidad en todo el mundo. En España, según el estudio IBERPOC, la prevalencia de EPOC es del 9.1\%, en edades comprendidas entre los 40 y los 70 años (5); pero, según el estudio EPI-SCAN (Epidemiologic Study of COPD in Spain), la prevalencia según los criterios GOLD es del 10.2\% (IC95\% 9,2-11,1) de la población entre 40 a 85 años. En América Latina los datos epidemiológicos son escasos. PLATINO (Proyecto Latinoamericano de Investigación en Obstrucción Pulmonar) arrojo los siguientes resultados de cinco ciudades latinoamericanas: el tabaquismo actual se presentó en $28 \%$ de los sujetos encuestados. Estos presentaron $47 \%$ más EPOC que los no fumadores; esta probabilidad fue $78 \%$ mayor para aquellos fumadores con un índice de paquetes/año mayor a 10.

Los resultados del estudio colombiano PREPOCOL señalan que EPOC tiene una prevalencia de $8.9 \%$ en mayores de 40 años, además que el cigarrillo es el principal condicionante de la enfermedad y que la exposición al humo de leña no está muy distante en su capacidad de generarla, en hombres y mujeres. El estudio ADEPOCLE, es un estudio epidemiológico, transversal, multicéntrico, no controlado en el ámbito de la atención primaria, en el que han participado 30 centros de salud de la provincia de León - España dio como resultado que el $86,67 \%$ de los pacientes con EPOC tenían antecedentes de tabaquismo. En estudio realizado en Guaranda - Ecuador, el $63.33 \%$ de los pacientes con EPOC pertenecen al grupo de fumadores activos; y el 12,24\% de la población eran Fumadores Pasivos. (8)

Otro estudio realizado en Ecuador en hospital Martin Icaza de Babahoyo identificaron al tabaquismo como factor de riesgo más importante para desarrollar EPOC, del $68 \%$ de los pacientes que son fumadores activos, el $58 \%$ ha desarrollado la EPOC y $42 \%$ son fumadores pasivos que en ocasiones ya han presentados signos y síntomas de la EPOC. (9)

En el desarrollo de la EPOC también interviene otros factores que cada vez son más importantes, como es la exposición a otras sustancias presentes en el aire inspirado, como es el caso de 
la exposición ocupacional. En el estudio National Health and Nutrition Examination Survey III (NHANES III), en los EE. UU, se constató que la exposición ocupacional puede ser causa de un $19 \%$ de los casos de EPOC, e incluso de un $32 \%$ de los casos en enfermos que nunca han fumado. El estudio platino realizado en el año 2016 en Santiago de Chile establecido la prevalencia de EPOC en mayores de 40 años, siendo fumadores el $48 \%$ y con exposición a combustión de biomasa un $61 \%$, en estudio realizado en Guaranda - Ecuador la situación edad varía, pues la prevalencia es mayor de 60 años con el $60,20 \%$, en cuanto a los fumadores la prevalencia es mayor con el $66,33 \%$, pero la exposición a combustión de biomasa es similar con el $71,4 \%$

El estudio realizado en la región de Maule-Chile en el 2017 proporciona el dato que la EPOC es más frecuente en los pacientes de entre 45-54 años, situación que es diferente a la encontrada en la población de Guaranda donde el grupo de edad que mayoritariamente fue afectado fue mayor de 60 años con el $60.20 \%$ estas diferencias pueden deberse a que los factores de exposición en son diferentes.

Medidas de prevención: Entre las medidas generales aplicadas destacan el no fumar $\mathrm{o}$ abandono del tabaco, nutrición adecuada, realización de actividad física regular, vacunación antigripal, vacunación antineumocócica en todos los pacientes y educación sanitaria de los pacientes para favorecer el autocuidado:

Ejercicio: El estudio ADEPOCLE indica que el $73,67 \%$ de los pacientes con EPOC realizaba ejercicio de forma regular según se los había recomendado su médico de familia y/o neumólogo. (10). La realización de ejercicio físico se asocia con un menor número de agudizaciones, menor gravedad, y menor número de hospitalizaciones. Un programa de entrenamiento muscular de 20 sesiones contribuye a una mejoría en la calidad de vida, la tolerancia al ejercicio y el pronóstico de los pacientes con EPOC con exacerbaciones leves o moderadas. La actividad física ha demostrado ser relevante en cuanto a la evolución y pronóstico de la enfermedad. Existen estudios que indican que cuanto mayor es la actividad física menor era la disminución de la función pulmonar, tanto en fumadores activos como en antiguos fumadores. Además, el ejercicio físico reduce el estrés oxidativo, tiene efecto antiinflamatorio, reduce la frecuencia de las infecciones de vías respiratorias.

Dieta equilibrada: En el estudio ADEPOCLE El $88,76 \%$ de los pacientes sigue una dieta equilibrada según las recomendaciones para pacientes con EPOC. A pesar de ello, la prevalencia de la malnutrición es alta en la EPOC. En la provincia de León, en menos del 5\% de los casos se utilizan suplementos dietéticos, especialmente en pacientes inmovilizados y caquécticos con EPOC. La evidencia indica que una dieta bien equilibrada es beneficiosa para todos los pacientes con EPOC, no solo por sus potenciales beneficios pulmonares, sino también por sus beneficios metabólicos y sobre el riesgo cardiovascular. Otros estudios han demostrado que la malnutrición condiciona un peor pronóstico, por tanto, la dieta y el ejercicio van de la mano en mejorar las condiciones de vida del paciente con EPOC

Vacunación antigripal. La vacunación antigripal es considerada la medida de prevención primaria más eficaz para prevenir la agudización de la EPOC (5). En algunos estudios la vacunación antigripal se ha asociado a la disminución en el número de ingresos hospitalarios por agudización de la enfermedad (6$8 \%$ ). El $80 \%$ de la mortalidad en estos pacientes se atribuye directamente a complicaciones de la gripe. La vacunación antigripal reduce la mortalidad en un $41 \%$ en los individuos de alto riesgo. La Guía GesEPOC recomienda la vacunación antigripal a todos los pacientes con EPOC, para evitar las exacerbaciones durante la época epidémica. A pesar de que esta vacuna está recomendada, como medida de prevención, las coberturas en algunos países son variadas alcanzando en algunos el $49 \%$ y en otros el $89 \%$. Además, hay que tener encuenta que únicamente el $18 \%$ de los pacientes vacunados lo solicitan directamente a su médico de atención primaria.

Vacunación antineumocócica. La infección neumocócica y en especial la enfermedad neumocócica invasiva (ENI) es una importante causa de morbilidad y mortalidad en el mundo. Los pacientes con EPOC tienen aumentado el riesgo de ENI y su evolución. Un consenso reciente sobre la vacunación antineumocócica en el adulto con patología de base recomienda en los pacientes inmunocompetentes con EPOC la administración de vacuna neumocócica conjugada de 13 serotipos (VNC13) en no vacunados y en los vacunados previamente con vacuna neumocócica polisacárida $(V N P)$ un año después. Los pacientes con mayor número de ingresos son los que menor tasa de vacunación antineumocócica presentan con cualquiera de las dos vacunas. (11)

La nueva vacuna neumocócica conjugada de 13 serotipos aporta una mayor respuesta inmunológica, incluso en mayores de 70 años que han sido previamente vacunados con la vacuna neumocócica polisacárida. Esto es especialmente relevante para la EPOC, ya que confiere un riesgo elevado de enfermedad neumocócica invasiva y la mayoría de pacientes son de edad avanzada. Por lo tanto, el grupo de trabajo del área de tabaquismo de la SEPAR recomienda la vacunación antineumocócica utilizando una sola dosis de $V N C 13$ en todos los fumadores, independientemente de su edad e intensidad y/o carga de consumo, que padecen enfermedades respiratorias como la EPOC. 
Las intervenciones de enfermería, están dirigidas tanto a la promoción de la salud, como a la prevención de la enfermedad como la EPOC, por lo tanto, el rol de la enfermera es la educación para favorecer el autocuidado de los pacientes y fomentar una vida más saludable, considerando la individualidad de cada paciente, pues cada caso tiene factores de riesgo y situaciones diferentes. Es de suma importancia que el personal de salud muestre empatía con el paciente, pues ello también incide en el autocuidado y la adherencia al tratamiento, además la educación debe abarcar al entorno familiar.

\section{Conclusiones}

Luego de haber realizado una revisión bibliográfica para identificar los factores de riesgo que involucra una EPOC se encontró, que los estudios realizados coinciden en: consumo excesivo de tabaco, personas que padecen de enfermedades respiratorias tales como el asma, fumadores pasivos, y personas que están expuestas a aires contaminados.

Entre las principales medidas preventivas para prevenir la EPOC o mejorar la calidad de vida de aquellos que ya la poseen están: evitar el consumo de tabaco, lugares donde se encuentren personas fumadoras y tratar de eludir lugares donde halla aire contaminado, vapores químicos y polvo, llevar una alimentación equilibrada y adecuada para personas con EPOC, realizar ejercicios de acuerdo a su estado de salud, recibir inmunización para enfermedades respiratorias.

La primordial intervención de enfermería es la educación sanitaria, por parte del personal de Enfermería, dirigido tanto al paciente como a su familia, indicar que debe abandonar el hábito del tabaco, evitar los contaminantes ambientales, prevenir las complicaciones, indicar el uso correcto de inhaladores, aplicar medicamentos y ayudar en técnicas de respiración, orientar sobre una alimentación y ejercicios adecuados, así como las vacunas que puede recibir. 


\section{Colaboradores de la Investigación:}

Diana Castillo Yánez; Bryan López Baque; Párraga Bravo Luis; Roxana Ponce Gómez; Carlos Salazar Plúas; Angie Solórzano Quiroz; María Zambrano Cedeño.

Estudiantes de la Carrera de enfermería de la Universidad Estatal del Sur de Manabí.

\section{Referencias Bibliográficas}

1. Cuidate Plus. [Online].; 2019 [cited 2020 agosto. Available from: https://cuidateplus.marca.com/enfermedades/respiratorias/epoc.html.

2. Clinic Barcelona. Hospital Universitario. [Online].; 2918 [cited 2020 agosto. Available from: https://www.clinicbarcelona.org/asistencia/enfermedades/enfermedad-pulmonar-obstructiva-cronicaepoc/vivir-con-la-enfermedad.

3. Basilio DMRG. E MItepoc WIKI. Glosario. [Online].; 2018 [cited 2020 Agosto. Available from: https://www.mitepocwiki.net/glosario/epidemiologia-fisiopatologia-la-epoc-2/.

4. RIMA red informática de medicina avanzada. [Online].; 2020 [cited 2020 agosto. Available from: https://www.rima.org/Noticias.aspx.

5. Serrano Poveda Jackie Helen scmp. Determinar la prevalencia de epoc diagnosticada por espirometria en los pacientes atendidos en el hospital general iess milagro periodo 2016-2017. Trabajo de titulación. Milagro. Universidad Estatal de Milagro. Facultad de ciencias de la salud, Repositorio UNEMI ; Junio 2018.

6. GuzmanVictor Hugo MECsGE. Diagnóstico y tratamiento de la enfermedad pulmonar obstructiva. 1st ed. Quito: Sociedad Ecuatoriana de neumología ; 2018.

7. wms-spanish-Pocket-Guide-GOLD-2017.pdf. Global initiative for chronic obstructive lung disease, inc.. [Online].; 2017 [cited 2020 agosto. Available from: https://goldcopd.org/wpcontent/uploads/2016/04/wms-spanish-Pocket-Guide-GOLD-2017.pdf.

8. Tercero Adriana. Prevalencia de enfermedad pulmonar obstructiva crónica en la unidad de medicina interna del Hospital Alfredo Noboa Montenegro. Proyecto de investigacion para titulacion. Ambato: UNIVERSIDAD REGIONAL AUTÓNOMA DE LOS ANDES, Repositorio; 2019.

9. Loor Chichande Yedarly Rmd. Oxigenoterapia Y Su Influencia En La Evolución de la Enfermedad Pulmonar Obstructiva Crónica en Adultos que asisten al area de observación Hospital Martín Icaza Babahoyo. Informe final del Proyecto De Investigación Para Titulacion. Babahoyo- Los Rios Ecuador : UNIVERSIDAD TÉCNICA DE BABAHOYO. , Repositorio; 2018.

10. De Abajo Larriba Ana Beatriz DRÁ. Estimación de actividades preventivas en pacientes con enfermedad pulmonar obstructiva crónica. Estudio ADEPOCLE. Scielo. Madrid - España. 2016 sep oct ; vol 33. ( $\left.\mathrm{N}^{\circ} 5\right)$. 